\title{
Effect of Chronic Consumption of Powdered Tobacco (Snuff) on Pain Sensation in Mice.
}

\author{
Aduema,W., ${ }^{1 *}$ Osim, E.E., ${ }^{2}$ Okorocha,A.E., ${ }^{3}$ Onwe P.E., ${ }^{3}$ Ogiji, E.D. ${ }^{4}$ Izunwanne \\ D.I. \\ ${ }^{1}$ Department of Human Physiology, Gregory University, Uturu, Abia State, Nigeria. \\ ${ }^{2}$ Department of Human Physiology, University of Calabar, Nigeria. \\ ${ }^{3}$ Department of Medical Physiology, Ebonyi State University, Abakaliki, Nigeria. \\ ${ }^{4}$ Department of Pharmacology/Therapeutics, Ebonyi State University, Abakaliki, Nigeria.
}

\begin{abstract}
Pain may be defined as an unpleasant sensory and emotional experience, associated with actual or potential tissue damage. The leaves have social and ethnomedical applications. Tobacco is used traditionally to treat arches, snake bites, bowel complains, convulsions, nervous ailment and to cure/heal the pain of toothache when chewed (Melson, 1944). Therefore this study was designed to investigate the effect of chronic consumption of powdered tobacco on pain sensation using two groups of Swiss mice (control and test) weighing $18 \mathrm{~g}-28 \mathrm{~g}$ $(n=15$ each).The control received $100 \mathrm{~g}$ of normal rodent chow, while the test group received $1 \mathrm{~g}$ of powdered tobacco in $99 \mathrm{~g}$ of rodent chow per day. Water was given ad libitum while daily food and water intake, as well as body weight changes, were monitored during the 31-day study. The tail flick and formalin tests were used to assess pain sensation. The results showed that the latency of tail flick did not differ between the test and control group. However, in the formalin test, the frequency of paw attention in both phases of the test was significantly lower $(P<0.05,0.01)$ compared to the control group. The duration and frequency of grooming $(P<0.05,0.01)$ and SAP $(P<0.01)$ were also significantly lower in the tobacco diet group compared to the control in the early phase, thus, showing decrease pain sensation. Therefore, chronic consumption of powdered tobacco diet may decrease pain sensation.
\end{abstract}

Keywords: Powdered tobacco, snuff, pain sensation, tail flick and grooming.

\section{Introduction}

Man's quest for survival has led him to discover many plants that are of medicinal value even before the advent of orthodox medicine. One of such plants is tobacco nicotiana commonly called tobacco. Tobacco is a native medicinal plant of the nightshade family that is cultivated for their leaves, which, when cured is smoked as cigars or cigarettes, chewed as chow and sniffed as snuff. Tobacco is hallucinogenic in high doses and a stimulant in low doses (Breen, 1985; Trefz, 1990).

As an agricultural product, tobacco is processed from the fresh leaves of the genus nicotiana. Tobacco has long been grown in America, Africa, Europe and Asia about 6,000 Bc (Koop, 1986). Tobacco is known and used throughout all quarters of the globe in two major forms: the smoked and the smokeless. The smoked tobacco contains nicotine,etc which combined gives rise to addictive stimulant and euphoria properties. The smokeless tobacco which has different native names in Nigeria and these include; 'Anwuru' (in Igbo), 'Taba' (in Hausa) and 'Uwong' (in Efik). Smokeless or spit tobacco comes in two different forms, snuff and chewing tobacco. Snuff, a fine grain tobacco that comes in cans or pouches. Chewing tobacco comes in pouches in the forms of long strands of tobacco that when used, are commonly called "plugs"” or "chew". Many people believe that using smokeless tobacco is safer than smoking it. This however, is not true because smokeless tobacco can have health effects amongst which are addiction to nicotine and leukoplakia (Dempsey, 2001).

Generally, tobacco (smoked and smokeless type) contains nicotine and this accounts for its anxiogenic effects. In addition to nicotine, tobacco is said to contain over nineteen known cancer causing chemicals such as nicotine and harmine to name but a few (Downs, 2008). Available literature suggest also that tobacco is a violent absolute poison which when introduced in a moderate quantity into the body system, can produce harmful effects. In fact, a small quantity of tobacco in the stomach can excite violent vomiting with other alarming symptoms like headache and nausea (Koop, 1986).

Considering the fact that the nervous system controls the rapid activity of the body including muscle movement, changing visceral events, anxiety/fear, pain, emotion, behaviors and even the rate of secretion of some endocrine glands (Guyton and Hall, 2006). In spite of the various measures of treatment and prevention of pain, as well as other behavioural changes, they still remain a major problem. Therefore, most researchers have resorted to traditional medicine to solve this problem. Since tobacco contain nicotine that is a central nervous system stimulant and affects behavioural parameters, such as pain (Benowitz, 1988).Therefore this research on 
tobacco is worthwhile for the public to know whether powdered tobacco will be beneficial or harmful in the above neurobehavioural parameter.

\section{Materials And Methods}

Experimental animals/grouping: Thirty Swiss white mice of both sexes weighing between (18-28g) and bred at the animal room of the Department of Human Physiology, University of Nigeria, Nsukka, were used for this study after approval by the College Ethical Committee of Abia State University. The animals were transported to the animal house of Department of Physiology, Abia State University, Uturu, Nigeria, were they were acclimatized under standard laboratory conditions and given free access to normal feed and clean drinking tap water. The animals were randomly assigned into two groups, control and a test group. The animals in the control group received normal feed (rodent chow) only; while the test group received mixed feed of $1 \mathrm{~g}$ powdered tobacco per every $99 \mathrm{~g}$ of rodent chow (1\% of tobacco diet) for 31 days. This is sequel to the fact that the determined LD50 for intraperitoneal administration of powdered tobacco was $1316.44 \mathrm{mg} / \mathrm{kg}$.

Experimental design: The tail flick and the formalin test were used to assess pain sensation in mice. The Tail flick nociceptive assay as developed by D'Amour and Smith (1941) was used to assess the effect of powdered tobacco on pain. Water was boiled to a steam at $100^{\circ} \mathrm{c}$, and a portion of this hot water was taken and some cold water was added to reduce the temperature of the hot water to $49^{\circ} \mathrm{c}$ in which the experiments were carried out. This temperature was constantly maintained by adding cold water when the temperature is above $49^{0} \mathrm{c}$ or by adding hot water when the temperature decreases below $49^{\circ} \mathrm{c}$ before each experiment was carried out. Thermometer was also constantly immersed into the hot water to ensure accurate temperature is maintained.

The mouse was restrained loosely, gently handled and care was taken to prevent bite. A stop watch was set and started exactly at the time when the tail of the mouse was immersed into the hot water at $49^{0} \mathrm{c}$. The stop watch was stopped exactly when the mouse flicked its tails from the hot water. This time was recorded as the latency of tail flick. The experiment was repeated after 1 hour for each mouse. The experiments were carried out on both the control and the test groups, after the experiment, the animals were returned to their metabolic cages thereafter. The experiment lasted for a day.

Also, the formalin test has been regarded as being a more satisfactory model of clinical pain than the hot plate tests (Abbott et al., 1981). Mice were carried into the room in their home cages. Each mouse was picked at the base of its tail and $0.2 \mathrm{ml}$ of $2.5 \%$ formalin was injected into the right hind paw of the mouse using a needle and syringe. The animal was placed in the observation box and observed for five (5) minutes. The animal was then returned to its cages and allowed for thirty (30) minutes before it was taken back to the observation box to be re-observed for another five (5) minutes. This procedure was repeated for each animal. The experiment lasted for a day and the behaviours' scored were; (1) frequency of paw lick and paw attention, (2) frequency and duration of grooming and (3) frequency of stretch attend posture.

Statistical Analysis: Data between the groups was analyzed by Student T-test and one-way analysis of variance (ANOVA) followed by Post-hoc using Sidak's multiple test. Data were presented as Mean \pm SEM and a "P" value less than 0.05 , was considered statistically significant.

\section{Results}

Frequency of grooming following formalin administration

The grooming frequency which is the number of time the animal spent licking or scratching itself while stationary in both the early and late phase of the formalin test by tobacco diet fed with mice and their control, shows that their values were $6.57 \pm 0.75 / 5 \mathrm{~min}$ (control) and $3.66 \pm 1.75 / 5 \mathrm{~min}$ (for tobacco fed mice) in the early phase. The grooming frequency of the tobacco fed mice was significantly lower compared to control in the early phase of the test $(\mathrm{P}<0.01)$ but insignificant at the late phase as shown in figure 1 .

\section{Duration of grooming in the formalin test}

The grooming duration which connotes the duration of time the animal spent licking itself while stationary shows that the tobacco group of mice in the early phase of the formalin test was $20.3 \pm 3.07 \mathrm{sec}$ and was significantly lower compared to the control with the value $29.67 \pm 3.92 \mathrm{sec}(\mathrm{P}<0.05)$. The grooming duration of the tobacco diet fed mice in the late phase was $34.48 \pm 4.25 \mathrm{sec}$ and was however not significantly different compared to the control value of $32.95 \pm 6.01 \mathrm{sec}$ (Figure 2).

\section{Frequency of stretch attend posture (SAP) following formalin administration}

The stretch attend posture is the frequency of time the animal demonstrates forward elongation of the head and shoulders followed by retraction to its original position. Our result showed that in the early phase of the formalin test by control mice and mice fed tobacco diet, there was a tremendous decrease in values $13.18 \pm 1.18 / 5 \mathrm{~min}$ 
(control) and $6.07 \pm 0.86 / 5 \mathrm{~min}$ (tobacco fed group). The frequency of SAP of the tobacco diet fed mice was significantly $(\mathrm{P}<0.01)$ lower compared to control. Whereas in the late phase of the formalin test, the values were $3.86 \pm 0.47 / 5 \mathrm{~min}$ (control) and $3.36 \pm 0.56 / 5 \mathrm{~min}$ (tobacco fed group). The frequency of SAP of the mice fed with tobacco was not significantly different compared to the control (Figure 3 ).

\section{Frequency of right hind paw attention following formalin administration}

The frequency of attention to the right hind paw injected formalin in the early phase of the test of the tobacco fed mice was significantly lower compared to control $(\mathrm{P}<0.01)$. The frequency values were $16.71 \pm 1.48 / 5 \mathrm{~min}$ for control and 10.2 $\pm 1.48 / 5 \mathrm{~min}$ for tobacco diet fed mice (Figure 3). The frequency of attention to the right hind paw of the tobacco fed mice in the late phase was also significantly lower $(\mathrm{P}<0.05)$ compared to control. The values were $5.62 \pm 0.58 / 5 \mathrm{~min}$ (control) and $3.55 \pm 0.62 / 5 \mathrm{~min}$ for tobacco diet fed group (Figure 4).

\section{Frequency of right hind paw lick following administration of formalin}

The number of times the animals (mice) in both groups' licks their hind paw of which formalin was injected were $18.28 \pm 1.43 / 5 \mathrm{~min}$ for the control group and $14.23 \pm 1.61 / 5 \mathrm{~min}$ for the mice fed tobacco diet in the early phase of the test and $2.5 \pm 0.5 / 5 \mathrm{~min}$ (control) and $2.0 \pm 0 / 5 \mathrm{~min}$ (tobacco fed diet) in the late phase. The frequency of right hind paw lick of the tobacco fed mice was not significantly different compared to the control though its value seems lower than the control in both phases (Figure 5).

\section{Latency tail flick}

The latency of tail flick, which indicate the time it takes for the mice to flick its tail from the warm water, showed that the values for control and tobacco diet fed mice were $2.92 \pm 0.54 \mathrm{sec}$ and $3.52 \pm 0.47 \mathrm{sec}$ respectively. The latency of flicks by the tobacco fed mice was not significantly different compared to that of the control (Figure 6).

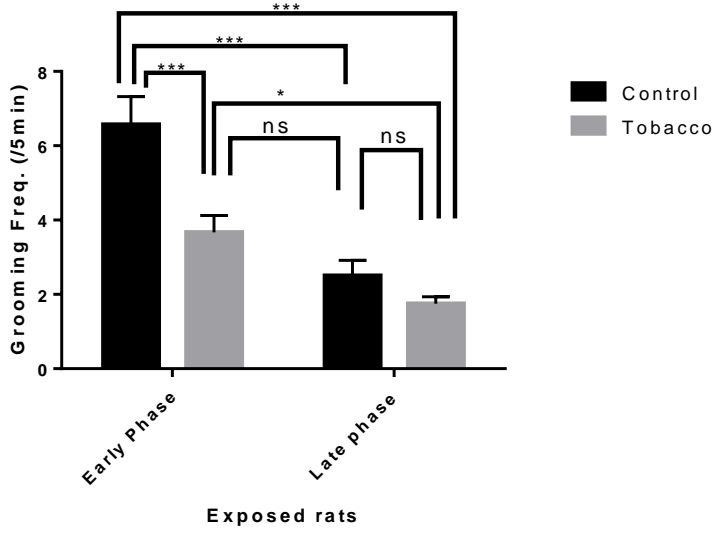

Figure 1 Comparison of frequency of grooming in the early and late phases of the formalin test between tobacco diets fed and control mice

Data were statistically analysed using Two-ways Anova with Sidak’s post hoc test.

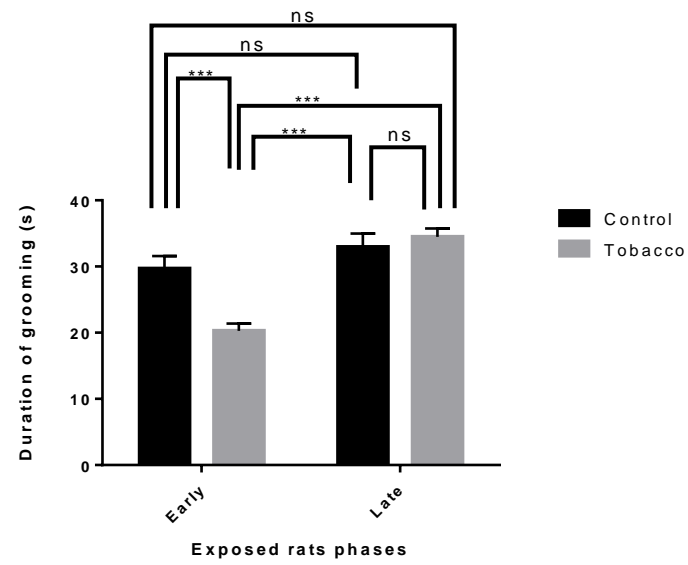

Figure 2 Comparison of duration of grooming in the early and late phases of the formalin test between tobacco diets fed and control mice. 
Data were statistically analysed using Two-ways Anova with Sidak’s post hoc test.

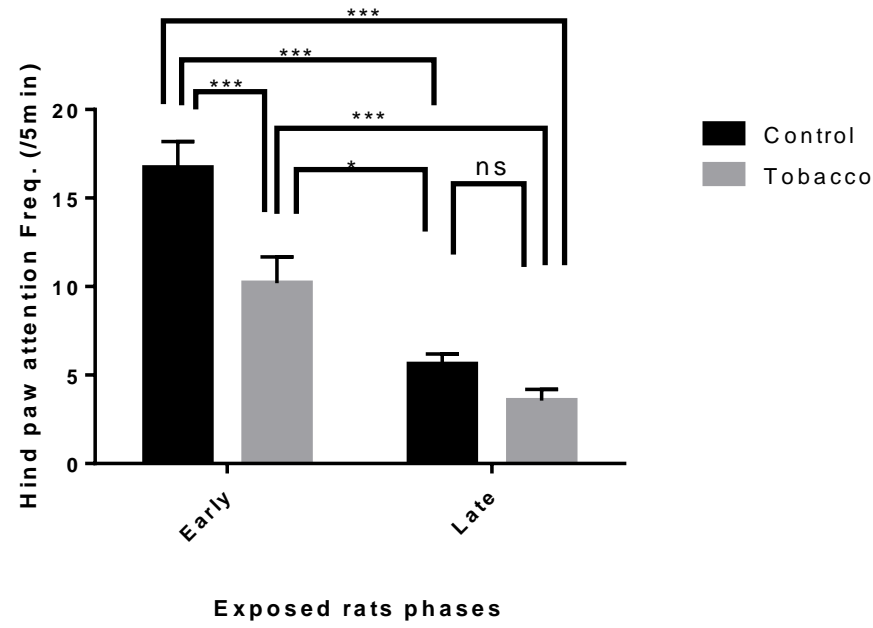

Figure 3 Comparison of frequency of hind paw attention in the early and late phases of the formalin test between tobacco diet fed and control mice.

Data were statistically analysed using Two-ways Anova with Sidak's post hoc test.

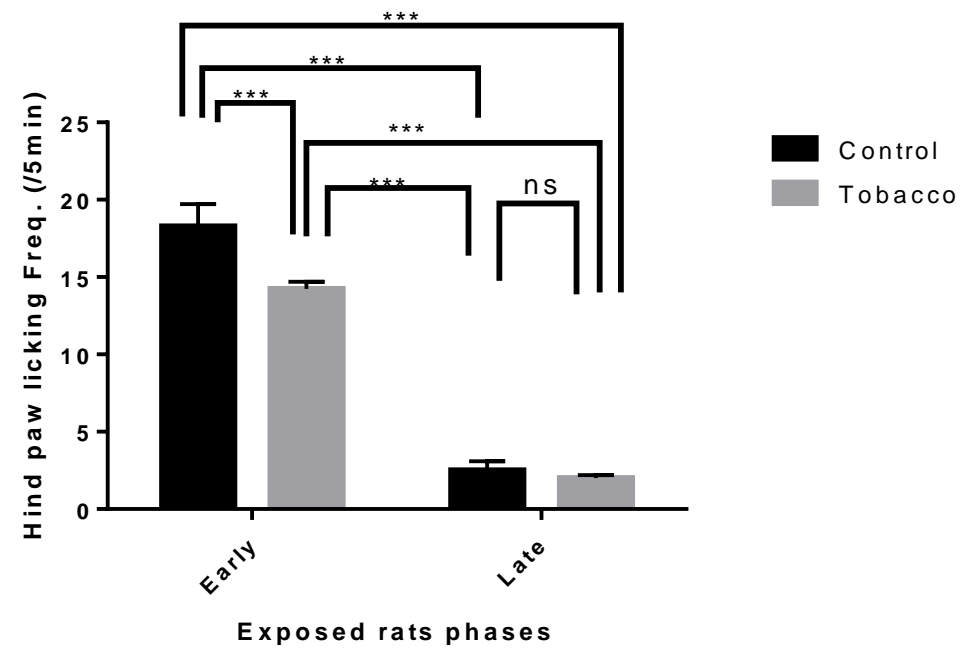

Figure 4 Comparison of frequency of hind paw lick in the early and late phases of the formalin test between tobacco diet fed and control mice.

Data were statistically analysed using Two-ways Anova with Sidak's post hoc test.

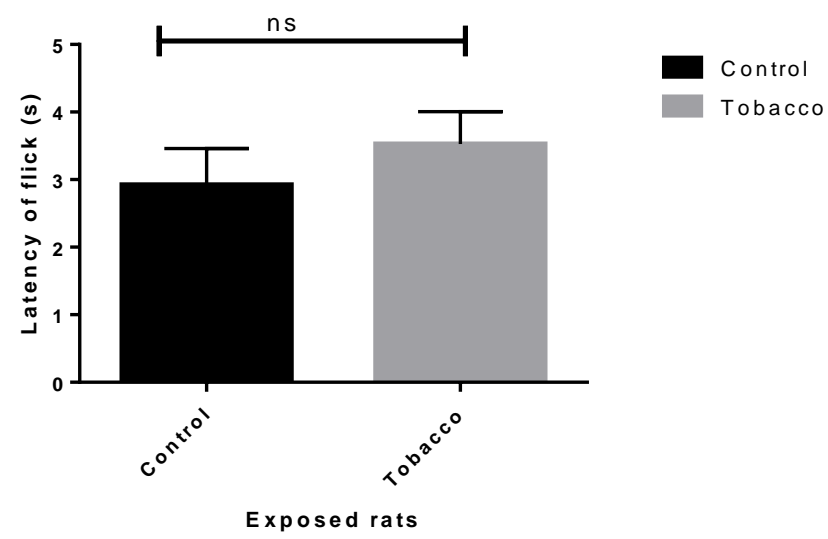

Figure 5 Comparison of latency of tail flick in the tail flick test between tobacco diet fed and control mice. 
Statistical relevance were obtained using Student t-test unpaired, which should that the latency of flick although longer in tobacco diet fed rat were not statistically significant $(\mathrm{P}<0.4065)$.

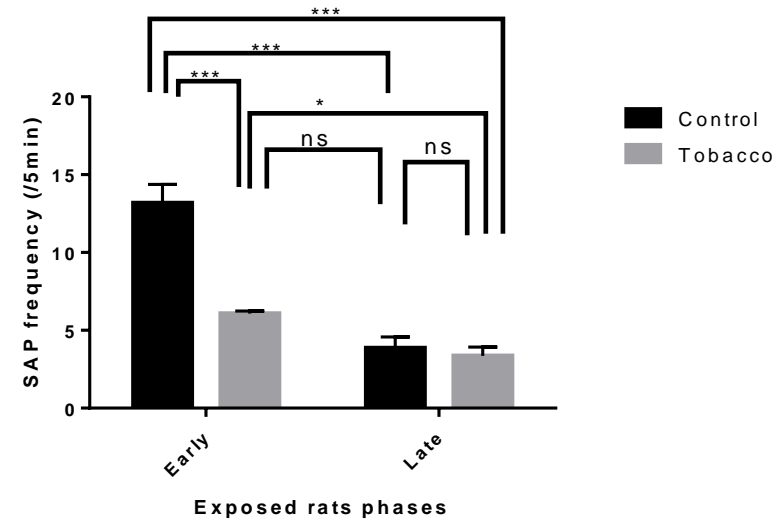

Figure 6 Comparison of frequency of stretch attend posture (SAP) in the early and late phases of the formalin test between the tobacco diets fed and control mice.

Data were statistically analysed using Two-ways Anova with Sidak’s post hoc test.

\section{Discussion And Conclusion}

In this study about pain sensation, the tail flick and formalin tests were employed. The principle is based on the inflammatory property of formalin and the reaction of the animal to hot water (Andrianbeloson, 2006). The tail flick is a system feature that involves when radiant heat is applied on the animal's tail - when the animal feels discomfort, there is a sudden tail movement (tail flick). This has proved that it is particularly a sensational property of pharmacological substance. It can also be used to evaluate basal thermal pain sensitivity or the study of putative genetic differences among animals without drugs. (Adrianbeloson, 2006). In the experiment, tail flick test showed no significant difference between both groups of mice. This result means that tobacco did not affect the spontaneous response to the sensation of pain. Formalin test is another model used in testing pain sensation in animals. Formalin generates an initial phase of activity (5-10 minutes, phase 1) and a second phase of activity (lasting 60-90 minutes, phase 2 or late phase) and this is seen with spontaneous behaviors. Inflammation contributes to phase 2 and the inter phase result from active inhibition. Formalin also results in tissue edema and this is longer lasting.

In the formalin experiment or test, the hind paw lick and attention were used to assess the changes in perception of pain sensation by the animal. Mice which feel more pain would exhibit more of these behaviors. The frequency of hind paw lick in both phases of the formalin test of the tobacco diet fed mice was not significantly different compared to the control. However, in the formalin test, the frequency of paw attention in both phases of the test was significantly lower in the test group compared to the control group. The duration and frequency of grooming and stretch attend posture was significantly lower in the tobacco diet group compared to the control in the early phase, thus, showing decrease pain sensation in the tobacco diet fed group compared to their control.

The report of Roefof and Perkins, (2004) showed a relationship between fear and pain-that decrease in fear would decrease pain and vice versa. The result is also consistent with decrease in fear related behavior such as stretch attends posture and grooming and decrease in pain sensation. This result suggests that the mice fed powdered tobacco (snuff) diet were less sensitive to pain compared to those fed with the control diet. The decrease in the sensitivity to pain caused by powdered tobacco may be attributed to the presence of flavonoid and phlobatannin in the powdered tobacco(snuff), which has been reported to reduce pain perception due to their anti-inflammatory properties (Hung-Xi et al., 2001).

In conclusion, chronic consumption of powdered tobacco diet decreases pain sensation. If this result is extrapolated to humans, long term consumption of tobacco diet may be beneficial in the control and management of pain in humans. Further research is however recommended to confirm if powdered tobacco (snuff) can be used as a pain killer.

\section{References}

[1]. Abbott, F.V., Franklin, K.B., Ludwick, R.J. and Melzack R. Apparent lack of tolerance in the formalin test suggests a different mechanism for morphine analgesia in different types of pain. Pharmacol. Biochem. Behav. 1981; 15: 637-40.

[2]. Adrianbeloson, R (2006). Evaluation Pain Sensitivity among animals.

[3]. Neuropsychopharmacology and Psychiatry, 24: 459-560. 
[4]. Alger, S.J., Maasch, S. N. and Riters, L.V (2009). Lesions to the media preoptic nucleus affect immediate early gene immunoglobulin in brain regions involved in song control and social behaviour in male European starlings. European Journal of Neuroscience, 45(6), 563-569.

[5]. Apkarian, A.V., Bushnell, M.C., Treede, R.D. and Zubieta, J.K (2005). Human brain mechanism of pain perception and regulation in health and disease. European Journal of pain, 9 (4), $463-84$.

[6]. Breen, T. H. (1965). Tobacco cloture: Princeton University Press. ISBN 0-691-00596-6. Source on tobacco culture in eighteenthcentury Virginia PP. 46-55.

[7]. D'Amour,F.E. and Smith,D.L(1941).A method for determining loss of pain sensation. Journal of Pharmacological and Experimental Therapeutics, 72, 74-79.

[8]. Delisa, and Catherine, F (1983). The nervous system and the body. American Journal of medical science. 239 (3), $302-304$

[9]. Demsey, A. K. (2001). Absorption of nicotinein cigarette and effect of cigar smoke. Nature, 226 (5252). $1231-1232$.

[10]. Down, F. (2008). Chemical Studies of Tobacco Smoke lxvii. Quantitative determination of alkaloids in tobacco by liquid chromatography. Journal of liquid chromatography, 3 (10): 1505 - 1515.

[11]. Farabee, G (2001). The central nervous system and behavior. New York, (2001 Pp. 450). International Journal of Psycho-analysis, 41:569.

[12]. Guyton,A.C. and Hall,J.E(2006).The nervous system. Textbook of Medical Physiology (pp.675-701).London: Saunders Company.

[13]. Hong-XI and Song, F. L (2001). Activity of plant flavenoids against antibiotic resident bacteria. Phythother. Res. 15: 39 - 43.

[14]. Ito, S.,Okuda - Ashiteka, E. and Minami, T (2001). Central and Peripheral roles of prostaglandins in pain and their interactions with novel neuropathies Nociceptin and nocistatin. Neuroscience Research, 41 (4). 299 - 332.

[15]. Kenny, P, J. Markoit, A. (2006). Nicotine Self administration acutely activates brain Reward systems and induces a long lasting increase in reward Sensitivity. Neuropsychopharmacology.31: 1203 -1211.

[16]. Kesangua, M. D (2007). Plant terpenoids; Application and future potential. International Journal of Ethnobiology and Ethnomedicine, 10: $16-19$.

[17]. King, S (2000). The classification and assessment of pain. International Review of Psychiatry, 12 (2), $86-90$.

[18]. Koop, .L. (1986). Mystery and History of Tobacco. Longman. New York.

[19]. Melson, (1944):'Ueber' das nicotin. Journal of Praktische Chemie., 32:372-377.

[20]. McCaffery, H(1968). "Pain psychogenesis and diagnosis". International review of psychiatry, 12(2), 99-102

[21]. Osim,E.E(2008).Neurophysiology(pp.24-27).Calabar.University of Calabar Press.

[22]. Roefof, P. and Perkins, K. A. (2004). Relationship between pain and fear. American Journal of Public Health; 70:420.

[23]. Terfz, L. D. (1990). Depending on tobacco. Time-life Publication, New York. 